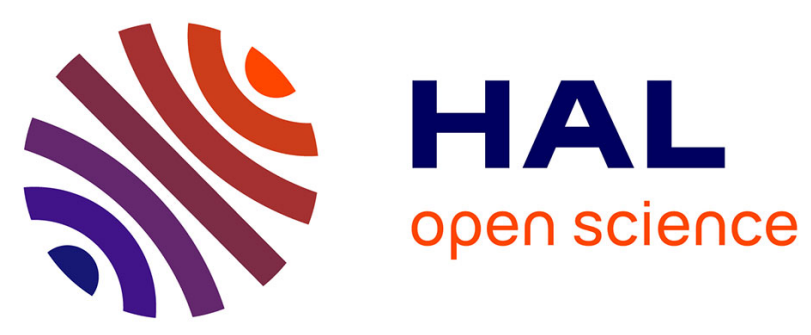

\title{
Nonlocal models for interface problems between dielectrics and metamaterials
}

Juan Pablo Borthagaray, Patrick Ciarlet

\section{To cite this version:}

Juan Pablo Borthagaray, Patrick Ciarlet. Nonlocal models for interface problems between dielectrics and metamaterials. 11th International Congress on Engineered Material Platforms for Novel Wave Phenomena - Metamaterials 2017, Aug 2017, Marseille, France. pp.3. hal-01547364

\section{HAL Id: hal-01547364 \\ https://hal.inria.fr/hal-01547364}

Submitted on 26 Jun 2017

HAL is a multi-disciplinary open access archive for the deposit and dissemination of scientific research documents, whether they are published or not. The documents may come from teaching and research institutions in France or abroad, or from public or private research centers.
L'archive ouverte pluridisciplinaire HAL, est destinée au dépôt et à la diffusion de documents scientifiques de niveau recherche, publiés ou non, émanant des établissements d'enseignement et de recherche français ou étrangers, des laboratoires publics ou privés. 


\title{
Nonlocal models for interface problems between dielectrics and metamaterials
}

\author{
Juan Pablo Borthagaray ${ }^{1}$ and Patrick Ciarlet $\mathrm{Jr}^{2}$ \\ ${ }^{1}$ IMAS-CONICET and Departamento de Matemática, FCEyN - Universidad de Buenos Aires, Ciudad \\ Universitaria, Pabellón I (1428) Buenos Aires, Argentina \\ ${ }^{2}$ POEMS (CNRS/ENSTA ParisTech/INRIA), 828, Bd des Maréchaux, 91762 Palaiseau Cedex, France \\ patrick.ciarlet@ensta-paristech.fr
}

\begin{abstract}
Consider two materials with permittivities/diffusivities of opposite sign, and separated by an interface with a corner. Then, when solving the classic (local) models derived from electromagnetics theory, strong singularities may appear. For instance the scalar problem may be ill-posed in $H^{1}$. To address this difficulty, we study here a nonlocal model for scalar problems with sign-changing coefficients. Numerical results indicate that the proposed nonlocal model has some key advantages over the local one.
\end{abstract}

\section{INTRODUCTION}

In electromagnetism, one can model materials that exhibit (almost) real-valued and strictly negative electric permittivity and/or magnetic permeability, within given frequency ranges. These so-called metamaterials, or lefthanded materials, raise unusual questions. Among others, proving the existence of electromagnetic fields, and computing them, is a challenging issue for a problem set in a domain $\Omega \subset \mathbf{R}^{n}(n=2,3)$ divided into a classical dielectric material and a metamaterial, when the frequency is in the above mentioned frequency range (see for instance $[11,12])$. The main issue is that the problem is ill-posed on some situations (see $\S$ II.), and as a consequence its numerical solution is unstable. To adress this difficulty, we propose to reformulate/transform the problem by using a nonlocal framework ( $($ III.), and then to study the numerical approximation of the nonlocal problem (§ IV.). Another approach would be to add a small, fixed imaginary part to the sign-changing coefficient that appears in the principal part of the PDE, in regions where it takes negative values [8].

\section{MODEL PROBLEM}

Let us consider a problem in a two-dimensional domain, set in the time-harmonic regime with pulsation $\omega>$ 0 . Then, the transmission problems in the transverse magnetic (TM) and transverse electric (TE) modes can be reduced to scalar problems like

$$
\operatorname{div}(\sigma \nabla u)+\omega^{2} \varsigma u=f \text { in } \Omega,
$$

with a source term $f$, and $(\sigma, \varsigma)$ equal to $\left(\epsilon^{-1}, \mu\right)$ or $\left(\mu^{-1}, \epsilon\right)$, where $\epsilon$ is the electric permeability, $\mu$ is the magnetic permeability, plus boundary conditions. The study of Maxwell's equation with sign-changing coefficients has been carried out in [3]-[4]-[7]. In particular, in [4], by resorting to the $T$-coercivity approach, it was shown that electric and magnetic Maxwell transmission problems are well-posed as soon as the associated three-dimensional scalar problems are well-posed. Thus, we focus on a model scalar problem.

Consider a partition $\bar{\Omega}=\overline{\Omega_{1}} \cup \overline{\Omega_{2}}$, with $\Omega_{1} \cap \Omega_{2}=\emptyset$ and $\Sigma=\partial \Omega_{1} \cap \partial \Omega_{2}$ the interface. We study a problem in which there is a dielectric material in $\Omega_{1}$ and an idealized metamaterial in $\Omega_{2}$. Namely, let $\sigma_{k} \in L^{\infty}\left(\Omega_{k}\right)$ ( $k=1,2$ ) be real-valued functions satisfying $\sigma_{1} \geq c_{1}>0$ a.e. in $\Omega_{1}$ and $\sigma_{2} \leq c_{2}<0$ a.e. in $\Omega_{2}$, where $c_{k}$ $(k=1,2)$ are constant numbers. Define the sign-changing $\sigma \in L^{\infty}(\Omega)$ by $\left.\sigma\right|_{\Omega_{k}}=\sigma_{k}$, and consider $\varsigma \in L^{\infty}(\Omega)$. We complement the PDE with a homogeneous Dirichlet boundary condition and assume that the right hand side function $f$ belongs to $L^{2}(\Omega)$. Thus, we look for solutions in the function space $H_{0}^{1}(\Omega)$, which is equipped with the usual energy-norm $\|v\|_{H_{0}^{1}(\Omega)}:=\left(\int_{\Omega}|\nabla v|^{2} d x\right)^{1 / 2}$. Due to the compactness of the embedding $H_{0}^{1}(\Omega) \subset L^{2}(\Omega)$, it is enough to consider the principal part of the PDE, $A u=-\operatorname{div}(\sigma \nabla u)$, and we are led to analyze mathematically the problem:

$$
\text { find } u \in H_{0}^{1}(\Omega) \text { such that }-\operatorname{div}(\sigma \nabla u)=f \text { in } \Omega .
$$


Above, the differential operators, namely the divergence and gradient operators, are local. Hence (1) may be called a local problem [10]. In variational form, it writes:

$$
\text { find } u \in H_{0}^{1}(\Omega) \text { s.t. } \forall v \in H_{0}^{1}(\Omega), \int_{\Omega} \sigma(x) \nabla u(x) \cdot \nabla v(x) d x=\int_{\Omega} f v d x .
$$

When $\sigma_{1}$ and $\sigma_{2}$ are constants, Costabel and Stephan [9] proved that, if the interface $\Sigma$ is of class $C^{2}$, then the operator $A$ is Fredholm if and only if the contrast $\kappa_{\sigma}=\sigma_{2} / \sigma_{1}$ is different from -1 . In general situations and independently of the geometry of $\Sigma$, problem (1) is of Fredholm type if and only if the contrast lies outside some interval $I_{c}$, called the critical interval, which always contains the value -1 .

The range of the critical interval depends on the interface. For example, Bonnet-Ben Dhia, Dauge and Ramdani [6] analyzed the effect of corners: they proved that in a square minus square geometry it holds that $I_{c}=$ $[-3,-1 / 3]$. Furthermore, the solution exhibits strong singularities at these regions when the contrast approaches the critical interval. The influence of corners at the interface between the two materials for problem (1) set in a particular geometry (with one corner of particular aperture) has been mathematically clarified in [5]. In such case, if the contrast lies inside the critical interval, Fredholm property is lost because of the existence of two strongly oscillating singularities at the corner, responsible for the ill-posedness in the classical framework. These singularities can be interpreted as waves propagating towards or outwards the corner. Then selecting the outgoing singularity by means of a limiting absorption principle allows to recover Fredholmness of the problem. A numerical method using Perfectly Matched Layers near the corner has then been derived, which gives satisfactory results [2].

\section{NONLOCAL MODEL PROBLEM}

We propose a method to shrink the critical interval for the contrast whenever the interface $\Sigma$ has corners, without having to cope with the singularities there. Mathematically, the ill-posedness of problem (1) means that the usual energy-norm is not adequate to measure the behavior of the solution near the interface. To address this difficulty, we choose to utilize norms able to capture the phenomena near the interface by means of introducing a nonlocal interaction model for the materials. Namely, we use the function space $\widetilde{H^{s}}(\Omega)=\left\{v \in H^{s}(\Omega)\right.$ s.t. $\left.\tilde{v} \in H^{s}\left(\mathbf{R}^{n}\right)\right\}$ where $\tilde{v}$ is the continuation of $v$ by 0 to $\mathbf{R}^{n} \backslash \Omega$, for some exponent $0<s<1$, equipped with the norm

$$
\|v\|_{\widetilde{H}^{s}(\Omega)}=\int_{\mathbf{R}^{n} \times \mathbf{R}^{n}} \frac{|\tilde{v}(x)-\tilde{v}(y)|^{2}}{|x-y|^{n+2 s}} d y d x .
$$

In essence, these norms are nonlocal. The nonlocal counterpart of the variational formulation (2) writes

$$
\text { find } u \in \widetilde{H}^{s}(\Omega) \text { s.t. } \forall v \in \widetilde{H}^{s}(\Omega), \int_{\mathbf{R}^{n} \times \mathbf{R}^{n}} \underline{\sigma}(x, y) \frac{(\tilde{u}(x)-\tilde{u}(y))(\tilde{v}(x)-\tilde{v}(y))}{|x-y|^{n+2 s}} d y d x=\int_{\Omega} f v d x \text {. }
$$

Assuming that $\sigma_{1}$ and $\sigma_{2}$ are constants, we propose to use

$$
\underline{\sigma}(x, y)= \begin{cases}\sigma_{1} & \text { if } x \in \Omega_{1}, y \in \Omega_{1} \cup \Omega^{c}, \\ \sigma_{2} & \text { if } x \in \Omega_{2}, y \in \Omega_{2} \cup \Omega^{c}, \\ \frac{1}{2}\left(\sigma_{1}+\sigma_{2}\right) & \text { if } x \in \Omega_{1}, y \in \Omega_{2} .\end{cases}
$$

These nonlocal norms are weaker than the usual energy-norm involved in the analysis of (2). So when considering (3), we are expanding the domain of the operator associated to the problem, while reducing its range. Hence, in this nonlocal framework, we expect that the operator remains of Fredholm type for a wider range of contrasts. The exponent $s$ accounts for the nonlocality of the problem: using an appropriate scaling, in the limit $s \rightarrow 1$ one recovers the local problem (1) almost everywhere.

\section{NUMERICAL EXPERIMENTS}

We use a conforming Finite Element Method to discretize the nonlocal problem (3), cf. [1]. The computations are carried out for two sample geometries:

Symmetric geometry: $\quad \Omega=(-1,1) \times(0,1), \quad \Omega_{1}=(-1,0) \times(0,1), \quad \Omega_{2}=(0,1)^{2}$;

Square minus square geometry: $\quad \Omega=(-1,1) \times(0,2), \quad \Omega_{1}=\operatorname{int}\left(\Omega \backslash \Omega_{2}\right), \Omega_{2}=(0,1)^{2}$. 
In $\Omega_{1}$, we fix $\sigma_{1}=1$, while $\sigma_{2}$ belongs to $(-\infty, 0)$. The right-hand side $f$ is equal to 1 over $\Omega$. In principle, the nonlocality exponent $s$ may span $(0,1)$. Figure 1 displays some of the outputs of these simulations.
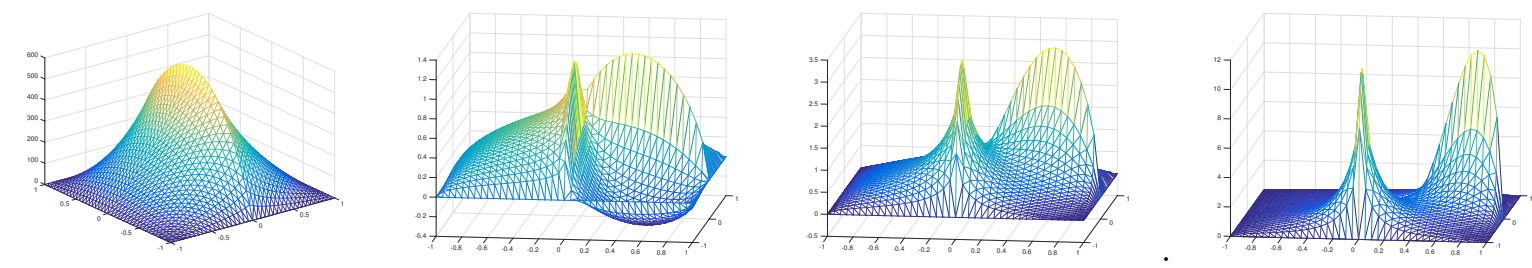

Fig. 1: Computational results for the nonlocal problem (3). First result: numerical solution for the symmetric geometry, with $s=0.999$ and $\sigma_{2}=-0.999$. Other results: numerical solutions for the square minus square geometry, with $s=0.7$. From left to right, $\sigma_{2}$ is taken equal to $\{-0.5,-0.9,-0.99\}$.

In the symmetric geometry, we are considering $s$ close to 1 , so that the nonlocal problem approaches the local one, and for the latter the critical interval reduces to $I_{c}=\{-1\}$. Regarding the problem set in the square minus square geometry, we checked that the solution is numerically stable for $\sigma_{2}=-0.5$, namely that it does not depend significantly on the meshize. Observe that for the corresponding local problem, this value lies within the critical interval. So, it seems that the nonlocal model shrinks the critical interval for the contrast. Increasing the value of $\left|\sigma_{2}\right|$ leads to larger amplitudes, as can be seen in the rightmost two figures.

\section{CONCLUSION}

For interface problems between dielectrics and metamaterials, it appears that nonlocal models may help derive acceptable numerical solutions, even when their local counterparts fail. The next step will be the numerical analysis of the nonlocal method, based on the $T$-coercivity theory.

\section{REFERENCES}

[1] G. Acosta, F.M. Bersetche and J.-P. Borthagaray, "A short FEM implementation for a 2D homogeneous Dirichlet problem of a fractional Laplacian," Computers and Mathematics with Applications (to appear).

[2] A.-S. Bonnet-Ben Dhia, C. Carvalho, L. Chesnel and P. Ciarlet Jr., "On the use of perfectly matched layers at corners for scattering problems with sign-changing coefficients," Journal of Computational Physics, vol. 322, pp. 224-247, 2016.

[3] A.-S. Bonnet-Ben Dhia, L. Chesnel and P. Ciarlet Jr., "Two-dimensional Maxwell's equations with sign-changing coefficients," Applied Numerical Mathematics, vol. 79, pp. 29-41, 2014.

[4] A.-S. Bonnet-Ben Dhia, L. Chesnel and P. Ciarlet Jr., "T-coercivity for the Maxwell problem with sign-changing coefficients," Communications in Partial Differential Equations, vol. 39, pp. 1007-1031, 2014.

[5] A.-S. Bonnet-Ben Dhia, L. Chesnel and X. Claeys, "Radiation condition for a non-smooth interface between a dielectric and a metamaterial," Mathematical Models and Methods in Applied Sciences, vol. 23, pp. 1629-1662, 2013.

[6] A.-S. Bonnet-Ben Dhia, M. Dauge and K. Ramdani, "Analyse spectrale et singularités d'un problème de transmission non coercif," Comptes-Rendus de l'Académie des Sciences, Série I - Mathématiques, vol. 328, pp. 717-720, 1999.

[7] S.C. Brenner, J. Gedicke and L.-Y. Sung, "Hodge decomposition for two-dimensional time-harmonic Maxwell's equations: impedance boundary condition," Mathematical Methods in the Applied Sciences, vol. 40, pp. 370-390, 2017.

[8] S. Chakrabarti, S.A. Ramakrishna and S. Guenneau, "Finite checkerboards of dissipative negative refractive index," Optics Express, vol. 14, pp. 12950-12957, 2006.

[9] M. Costabel and E. Stephan, "A direct boundary integral equation method for transmission problems," Journal of Mathematical Analysis and Applications, vol. 106, pp. 367-413, 1985.

[10] Q. Du, M. Gunzburger, R.B. Lehoucq, and K. Zhou, "A Nonlocal Vector Calculus, nonlocal volume-constrained problems, and nonlocal balance laws," Mathematical Models and Methods in Applied Sciences, vol. 23, pp. 493-540, 2013.

[11] P. Fernandes and M. Raffetto, "Well-posedness and finite element approximability of time-harmonic electromagnetic boundary value problems involving bianisotropic materials and metamaterials," Mathematical Models and Methods in Applied Sciences, vol. 19, pp. 2299-2335, 2009.

[12] H. Wallén, H. Kettunen and A. Sihvola, "Surface modes of negative-parameter interfaces and the importance of rounding sharp corners," Metamaterials, vol. 2, pp. 113-121, 2008. 